\title{
Correction: phase I study of neoadjuvant chemoradiotherapy with S-1 plus biweekly cisplatin for advanced gastric cancer patients with lymph node metastasis -KOGC04-
}

\author{
Satoru Matsuda', Tsunehiro Takahashi ${ }^{*}$, Junichi Fukada ${ }^{2}$, Kazumasa Fukuda ${ }^{1}$, Hirofumi Kawakubo', \\ Yoshiro Saikawa ${ }^{1}$, Osamu Kawaguchi ${ }^{2}$, Hiroya Takeuchi ${ }^{1}$, Naoyuki Shigematsu ${ }^{2}$ and Yuko Kitagawa ${ }^{1}$
}

\section{Correction}

After the publication of this work [1], we noted that owing to an inadvertent mistake, the description of the chemotherapy schedule shown in the Methods was different from that shown in the Abstract and the duration of S-1 treatment in the initial chemoradiotherapy was inadequately described in Figure 1. Therefore, Methods and Figure 1 were modified accordingly.

In the "Chemoradiotherapy" section in the Methods, the 1st and 3rd paragraphs should read as follows:

"The chemoradiotherapy protocol consisted of administration of S-1 plus biweekly cisplatin and radiation (Figure 1). The initial chemoradiotherapy schedule was for 6 weeks: S-1 was orally administered twice daily from the evening of day 1 to the morning of day 15 , and the total dose was based on the patient's body surface area
(BSA), as follows: $<1.25 \mathrm{~m}^{2}, 80 \mathrm{mg} ; 1.25-1.5 \mathrm{~m}^{2}$, $100 \mathrm{mg}$; and $>1.5 \mathrm{~m}^{2}, 120 \mathrm{mg}$. An escalating dose of cisplatin was administered by infusion over $1 \mathrm{~h}$ on days 1 and 15 without infusional hydration. The starting dose (level 1) of cisplatin was $15 \mathrm{mg} / \mathrm{m}^{2}$, the second dose (level 2) was $20 \mathrm{mg} / \mathrm{m}^{2}$, and the third dose (level 3) was $25 \mathrm{mg} / \mathrm{m}^{2}$."

"After initial chemoradiotherapy, one cycle of combination chemotherapy with S-1 plus biweekly cisplatin was delivered. This consisted of 42 days of S-1 administered from the evening of day 1 to the morning of day 29 and of cisplatin administered on days 1,15 , and 29."

In "Figure 1," based on the description in the manuscript, the duration of S-1 treatment in the initial chemoradiotherapy was revised to 1-15 days from 1-22 days (Figure 1).
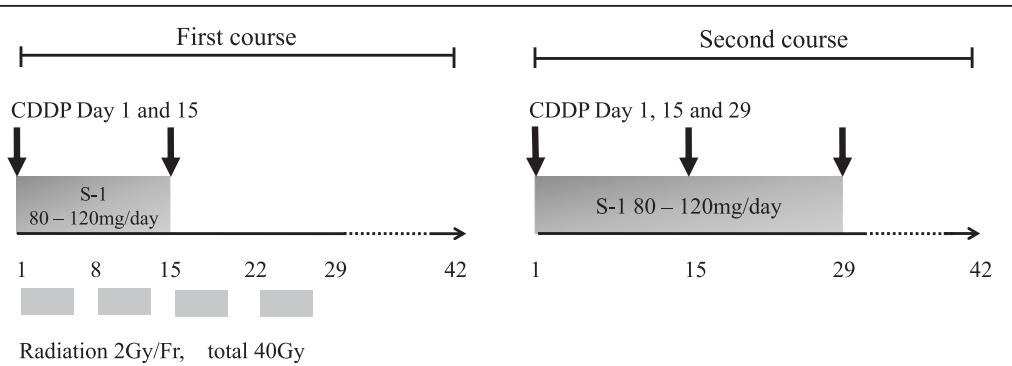

Figure 1 Chemoradiotherapy consisted of combination chemotherapy with S-1, biweekly cisplatin, and fractionated radiation therapy.

\footnotetext{
* Correspondence: surgeontsune@yahoo.co.jp

${ }^{1}$ Department of Surgery, Keio University School of Medicine, 35

Shinanomachi, Shinjuku-ku, Tokyo 160-8582, Japan

Full list of author information is available at the end of the article
} 


\section{Author details}

'Department of Surgery, Keio University School of Medicine, 35

Shinanomachi, Shinjuku-ku, Tokyo 160-8582, Japan. ${ }^{2}$ Department of

Radiology, Keio University School of Medicine, 35 Shinanomachi, Shinjuku-ku,

Tokyo 160-8582, Japan.

Received: 16 June 2014 Accepted: 16 June 2014

Published: 25 June 2014

\section{Reference}

1. Matsuda S, Takahashi T, Fukada J, Fukuda K, Kawakubo H, Saikawa Y,

Kawaguchi O, Takeuchi H, Shigematsu N, Kitagawa Y: Phase I study of neoadjuvant chemoradiotherapy with S-1 plus biweekly cisplatin for advanced gastric cancer patients with lymph node metastasis: -KOGC04. Radiat Oncol 2014, 9:9.

doi:10.1186/1748-717X-9-140

Cite this article as: Matsuda et al:: Correction: phase I study of neoadjuvant chemoradiotherapy with S-1 plus biweekly cisplatin for advanced gastric cancer patients with lymph node metastasis -KOGC04-. Radiation Oncology 2014 9:140.

\section{Submit your next manuscript to BioMed Central and take full advantage of:}

- Convenient online submission

- Thorough peer review

- No space constraints or color figure charges

- Immediate publication on acceptance

- Inclusion in PubMed, CAS, Scopus and Google Scholar

- Research which is freely available for redistribution 